\title{
Search for new Physics in the Dijet Mass Spectrum and Dijet Ratio in pp Collisions at $\sqrt{s}=7 \mathrm{TeV}$
}

\author{
CMS Collaboration \\ Konstantinos Kousouris* \\ Fermi National Accelerator Laboratory \\ E-mail: Konstantinos.Kousourisecern.ch
}

\begin{abstract}
A search for new Physics is performed with inclusive dijet final states in pp collisions, using data corresponding to an integrated luminosity of $120 \pm 13 \mathrm{nb}^{-1}$ collected by the CMS experiment at LHC. Generic upper limits at the $95 \%$ confidence level (CL) are presented on the product of the resonance cross section, branching fraction into dijets, and acceptance, separately for decays into quark-quark, quark-gluon, or gluon-gluon pairs. The data exclude new particles predicted in the following models at the 95\% CL: string resonances, with mass less than $1.67 \mathrm{TeV}$, excited quarks, with mass less than $0.59 \mathrm{TeV}$ and axigluons and colorons with mass less than $0.52 \mathrm{TeV}$. A search for quark compositeness in the form of quark contact interactions is conducted using the dijet centrality ratio, which quantifies the angular distribution of the dijets. The measurement is found to agree with the predictions of the Standard Model and the statistical analysis of the data provides a lower limit on the energy scale of quark contact interactions of $1.9 \mathrm{TeV}$ at the $95 \%$ confidence level. The above results extend previously published limits on these models.
\end{abstract}

35th International Conference of High Energy Physics - ICHEP2010,

July 22-28, 2010

Paris France

${ }^{*}$ Speaker. 


\section{Introduction}

Within the Standard Model, events with two energetic jets (dijets) arise in proton-proton collisions from parton-parton scattering. The dijet mass spectrum predicted by Quantum Chromodynamics (QCD) falls smoothly and steeply with increasing dijet mass. Many extensions of the Standard Model predict the existence of new massive objects that couple to quarks $(q)$ and gluons $(g)$, and result in resonant structures in the dijet mass spectrum. In addition, the angular distribution of jet pairs produced in the context of various new physics scenarios can help distinguish these mechanisms from the SM. New Physics beyond the SM typically produces more isotropic angular distributions than those predicted by QCD, resulting in higher dijet rate at lower absolute values of pseudorapidity. In this paper we report a search for narrow resonances in the dijet mass spectrum, and a search for contact interactions with the dijet centrality ratio. The data analyzed in this paper were collected in 2010 with the Compact Muon Solenoid (CMS) detector [1] at the CERN Large Hadron Collider, at a proton-proton collision energy of $\sqrt{s}=7 \mathrm{TeV}$, corresponding to an integrated luminosity of $120 \pm 13 \mathrm{nb}^{-1}$. A detailed description of the jet reconstruction, calibration and quality requirements can be found in [2].

\section{Search for Dijet Resonances in the Dijet Mass Spectrum}

The shape of the dijet mass distribution of the two leading jets is first compared with QCD predictions from the simulation. Then, a smooth parameterization of the dijet mass data distribution is used to model the background prediction for the narrow resonance search. The dijet resonance shapes for generic di-parton resonances containing $q q, q g$ and $g g$ partons were simulated in the limit that the natural widths are negligible compared to the experimental resolution of the dijet mass measurement. If a dijet resonance exists, it should appear in the dijet mass spectrum as a resonance peak on top of a smooth background. The measured dijet mass spectrum is shown in Fig. 1 (left) and the data are compared to the Monte Carlo prediction. Figure 1 (right) shows the dijet mass spectrum compared to a parameterized fit to a smooth function. The parameterization chosen is $\frac{d \sigma}{d m}=\frac{P_{0} \cdot(1-m / \sqrt{s})^{P_{1}}}{m^{P_{2}}}$. There is no indication for the presence of peaks above the background fit at the current level of data statistics. In the absence of a new Physics signal, we proceed to set upper limits, using a Bayesian formalism with uniform prior for the cross section [2]. A typical resonance shape for resonance mass of $0.7 \mathrm{TeV}$ is shown in Fig. 2 (left). The upper limits at 95\% C.L. set on the cross section times branching ratio of centrally $(|\eta|<1.3)$ produced dijet mass resonances are shown in Fig. 2 (right). Separate limits are reported for dijets with three different parton contents, quark-quark $(q q)$, quark-gluon $(q g)$ and gluon-gluon $(g g)$, in the final-state dijet system due to their differing resonance shapes. The limits are compared with calculations of the cross section times branching ratio for dijets in the fiducial acceptance $|\eta|<1.3$ from seven different models. The cross section for string resonances, which decay predominantly to $q g$, can be compared to the upper limit on the cross section for $q g$ resonances in Fig. 2 (right). We exclude at 95\% C.L. string resonances with mass less than $1.67 \mathrm{TeV}$, excited quarks with mass less than $0.59 \mathrm{TeV}$ and axigluons and colorons of mass less than $0.52 \mathrm{TeV}$. 

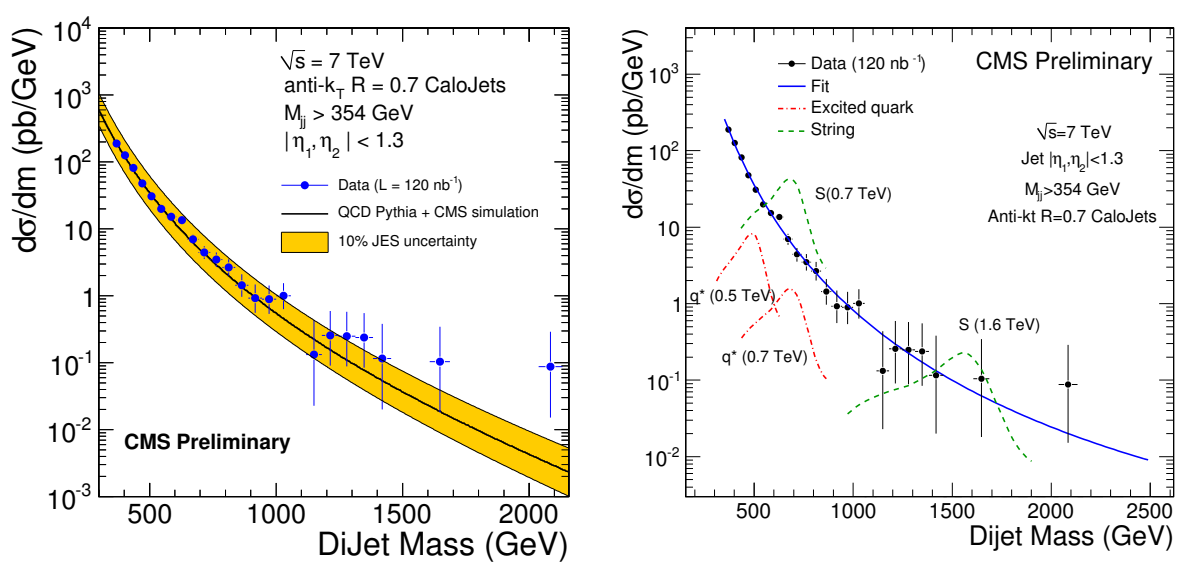

Figure 1: Left: the measured differential cross section (points) in dijet mass is compared to a QCD MC prediction. Right: the dijet mass distribution compared to simulations of excited quarks and string resonance signals in the CMS detector.
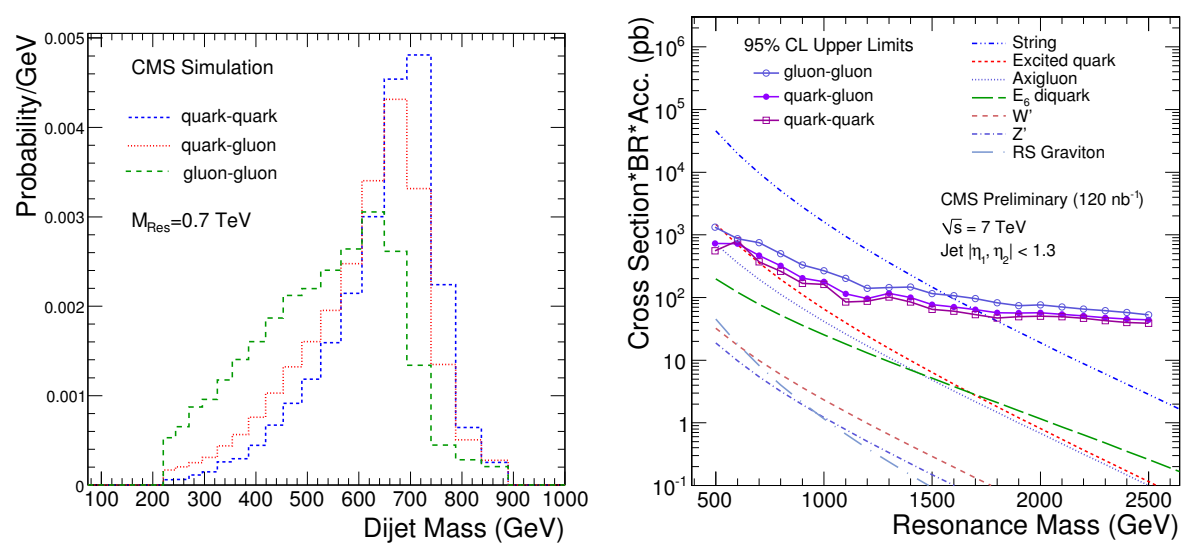

Figure 2: Left: simulated signal shapes of dijet mass resonances for $q \bar{q}(q q), q g$ and $g g$ resonances of mass 0.7 TeV. Right: the $95 \%$ C.L. upper limits on the cross section for dijet resonances compared to cross section predictions for several new physics models.

\section{Search for Contact Interactions with the Dijet Ratio}

The dijet centrality ratio is defined as $N(|\eta|<0.7) / N(0.7<|\eta|<1.3)$, which is the number of events with both jets in the region $|\eta|<0.7$ divided by the number of events with both jets in the region $0.7<|\eta|<1.3$. The dijet ratio provides a precise test of QCD and is sensitive to new physics. The measured dijet centrality ratio is compared to QCD perturbative calculations at nextto-leading order (NLO) [3]. In the SM the dijet centrality ratio is nearly flat as a function of dijet mass, with a value of $0.5-0.6$. In the presence of contact interaction, the dijet centrality ration rises rapidly with the departure from the SM prediction occurring at a dijet mass that depends on the scale $\Lambda$ of the interaction. Resonances such as excited quarks cause the ratio to peak near the mass of the resonance. The measured ratio is used here to search for contact interaction among left-handed quarks at an energy scale $\Lambda$ in the process $q q \rightarrow q q$. This is modeled with the effective 
Lagrangian $L_{q q}=\left( \pm 2 \pi / \Lambda^{2}\right)\left(\bar{q}_{L} \gamma^{\mu} q_{L}\right)\left(\bar{q}_{L} \gamma_{\mu} q_{L}\right)$ with " + " chosen for the sign of the interference.

Figure 3 (left) shows a comparison of the measured dijet centrality ratio with the predictions of NLO QCD and various new physics models. To quantitatively test for the presence of new physics in the dijet centrality ratio, we use a log-likelihood-ratio statistic $\left(\mathscr{R}_{L L}\right)$ that compares the null hypothesis (SM only) to the hypothesis that new physics effects are present in addition to the SM. We compare the value of $\mathscr{R}_{L L}$ in the data to distributions of the expected values for both hypotheses, obtained in ensembles of pseudo-experiments. In Fig. 3 (right) we show the $\mathscr{R}_{L L}$ for the data, the 95\% $\mathrm{CL}_{s}$ points, and the $\mathrm{SM}$ expectation (with 1 and $2 \sigma$ bands) versus contact interaction scale $\Lambda$. The limit is the point where the data line crosses the $95 \% \mathrm{CL}_{s}$ line and we exclude a contact interaction with scale $\Lambda<1.9 \mathrm{TeV}$ at $95 \%$ C.L.
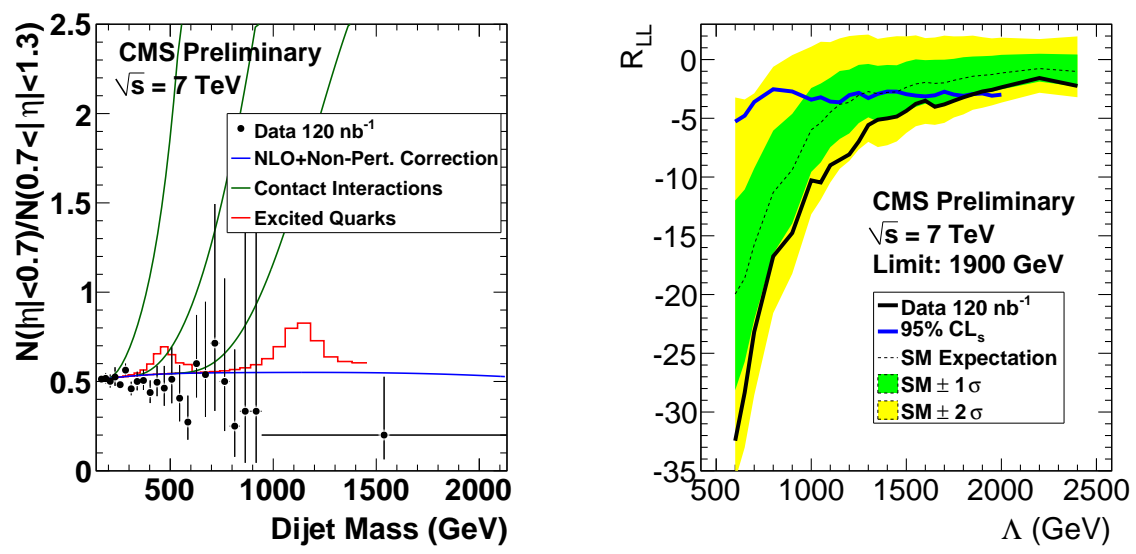

Figure 3: Left: dijet centrality ratio compared to QCD prediction, contact interactions with $\Lambda=500,1000$, and $1500 \mathrm{GeV}$, and excited quark resonances with masses of 500 and $1200 \mathrm{GeV}$. Right: limit summary plot for contact interaction scale $\Lambda$.

\section{Conclusions}

A search for narrow resonances and quark contact interaction has been performed with the CMS experiment in the inclusive dijet final state using $120 \pm 13 \mathrm{nb}^{-1}$ of integrated luminosity taken at a center-of-mass of $7 \mathrm{TeV}$ in proton-proton collisions at the LHC. The data are in agreement with the predictions of the Standard Model. We set upper limits at 95\% C.L. on the cross section times branching ratio of centrally $(|\eta|<1.3)$ produced dijet mass resonances and exclude a contact interaction with scale $\Lambda<1.9 \mathrm{TeV}$.

\section{References}

[1] CMS Collaboration, The CMS experiment at the CERN LHC, JINST 3 S08004, 2008.

[2] CMS Collaboration, Search for Dijet Resonances in the Dijet Mass Distribution in pp Collisions at $\sqrt{s}=7 \mathrm{TeV}$, CMS Physics Analysis Summary EXO-10-001, 2010.

[3] CMS Collaboration, Search for New Physics with the Dijet Centrality Ratio, CMS Physics Analysis Summary EXO-10-002, 2010. 